\title{
Fistula Vesicovaginal por Litíase: Relato de Caso
}

\author{
Vesicovaginal Fístula Caused by Lithiasis: A Case Report
}

Antônio Chambô Filho, Renata Costa Alves Kunsche, Valéria Lira Devens, Rolney Scardini, Fábio Leal Laignier Borges

\section{RESUMO}

As fístulas de etiologia traumática são raras, sobretudo as intrinsecas por litíase vesical. $O$ manejo dessas fistulas tem controvérsias no que diz respeito à técnica cirúrgica ideal. Uma variedade de tratamentos tem sido descrita, incluindo reparação cirúrgica por vias transvaginal e transabdominal. Os autores relatam o caso de uma paciente com queixa de perda urinária continua com evolução de seis meses, sendo identificado ao exame especular orificio fistuloso no terço médio da parede anterior da vagina, por onde se exteriorizava urina. Nesta topografia, identificava-se, durante o toque bimanual, estrutura de consistência pétrea. A suspeita de litiase vesical foi confirmada pela radiografia de pelve. O tratamento cirúrgico se deu em dois tempos, com exérese do cálculo vesical e posterior correção da fístula por via vaginal.

\section{PALAVRAS-CHAVE: Fístula. Fístula vesicovaginal. Cálculo vesical.}

\section{Introdução}

A abertura traumática entre o trato urinário e o meio externo é denominada fístula. Em todo mundo, a causa mais comum é o trabalho de parto obstruído ${ }^{1}$. O útero e a vagina são segmentos do canal genital que, em geral, participam da formação das fístulas urogenitais, predominando a variedade vesicovaginal ${ }^{2}$.

É provável que as fístulas vesicais em tocoginecologia existam desde que o homem assumiu sua postura ortostática, sendo o caso mais antigo até agora conhecido o descrito por Derry no Egito em $2050 \mathrm{aC}^{3}$. Apesar da escassez de dados estatísticos precisos, sabe-se que as fístulas urogenitais são freqüentes no Brasil ${ }^{2}$.

As fistulas constituem um dos grandes problemas ginecológicos, tanto pela complexidade de que se reveste seu tratamento, como pelos transtornos que as lesões fistulosas acarretam à paciente, em decorrência do escoamento incontrolável de urina através da vagina, responsável pela umidade e irritação constantes de seus genitais. A enfermidade também reflete-se no relacionamento social e familiar do doente. Devido

Corresponência:

Antônio Chambô Filho

Rua Constante Sodré $\mathrm{n}^{\circ}$ 1027, apt 301 - Praia do Canto 29055-420 - Vitoria - ES ao odor fétido que exala, o convivio com a fistulosa torna-se constrangedor para ela própria e mesmo para os mais intimos, levando-a conseqüentemente ao isolamento voluntário ou resultante do abandono até pelo próprio companheiro ${ }^{2}$.

Quanto à etiologia, as fistulas podem ser classificadas em obstétricas, cirúrgicas, neoplásicas, urológicas, traumáticas, actínicas, inflamatórias e congênitas ${ }^{2}$. As fístulas vesicovaginais traumáticas são raras. Counseller e Welch ${ }^{4}$ encontraram o trauma como fator etiológico em $1,7 \%$ das fistulas geniturinárias.

Santos ${ }^{5}$, em estudo que incluía 351 casos de fistulas urogenitais atendidos na clínica ginecológica do Hospital Getúlio Vargas da Universidade Federal do Piauí (Teresina) num espaço de 31 anos, incluindo 190 casos publicados em monografia anterior, observou apenas 2 casos $(0,6 \%)$ de fistulas traumáticas, um por cálculo vesical e um por empalação.

As fistulas traumáticas podem ser divididas para fins didáticos em extrínsecas e intrínsecas. As extrinsecas podem ser causadas por cateterismo vesical, queda a cavaleiro (empalação), acidente de trânsito com fratura de bacia, lesões por arma branca ou projétil de arma de fogo, instilação de líquido corrosivo na vagina, entre outros. As fistulas traumáticas intrínsecas quase sempre são decorrentes de cálculos vesicais, que formam um 
pertuito através da parede vesical ou da uretra em direção à vagina por compressão e necrose, e geralmente estão associadas a infecção de repetição do trato urinário ${ }^{2}$.

A maioria das fistulas vesicovaginais são fáceis de diagnosticar e classicamente apresentam-se como perda urinária diurna e noturna. As manobras diagnósticas que podem auxiliar na demonstração de uma pequena fístula em situações duvidosas incluem instilação de azul de metileno na bexiga com a colocação de tampão vaginal ou infusão de dióxido de carbono na vagina, o que induz à formação de bolhas quando se realiza cistoscopia. Durante a uretrocistoscopia com vaginoscopia, o tamanho e a posição exata da fistula em relação aos óstios ureterais devem ser bem documentados ${ }^{6}$.

O manejo de fistulas vesicovaginais representa um ponto de controvérsia no que diz respeito à técnica cirúrgica ideal. Uma grande variedade de tratamentos foi descrita, incluindo reparação cirúrgica por vias transvaginal e transabdominal ${ }^{7}$.

\section{Relato do Caso}

Paciente DPJ, 37 anos, procurou ambulatório de cirurgia ginecológica da Santa Casa de Misericórdia de Vitória no dia 21 de dezembro de 1998 , em bom estado geral, porém fragilizada psicologicamente, relatando perda de urina contínua com evolução de 6 meses, associada a dor no baixo ventre, disúria e transtorno no convivio social e familiar. Negava doenças ou cirurgias anteriores. Entre os antecedentes ginecológicos, relatava ciclos menstruais regulares, sendo o último parto transpélvico há 14 anos.

Ao exame físico a paciente apresentava-se em regular estado geral, corada e hidratada, com PA de 130/70 mmHg. Ao exame ginecológico observava-se vulva hiperemiada e com odor amoniacal e vagina úmida. Ao introduzir espéculo identificou-se presença de urina em moderada quantidade na vagina, sendo identificado no terço médio de sua parede anterior um orificio fistuloso. Ao exame de toque bimanual identificou-se estrutura de consistência endurecida (pétrea) na topografia da fistula, comprimindo-a.

Após internação foi realizado o teste com azul de metileno que confirmou a presença de fistula vesicovaginal. Foram solicitados exames pré-operatórios de rotina, que vieram sem alterações, com exceção do exame de sedimento urinário e urocultura que comprovaram infecção urinária (Escherichia coli sensivel a norfloxacina), e tam- bém da radiografia de abdome/pelve, que evidenciou em topografia vesical uma imagem radiopaca sugerindo litíase vesical (Figura 1).

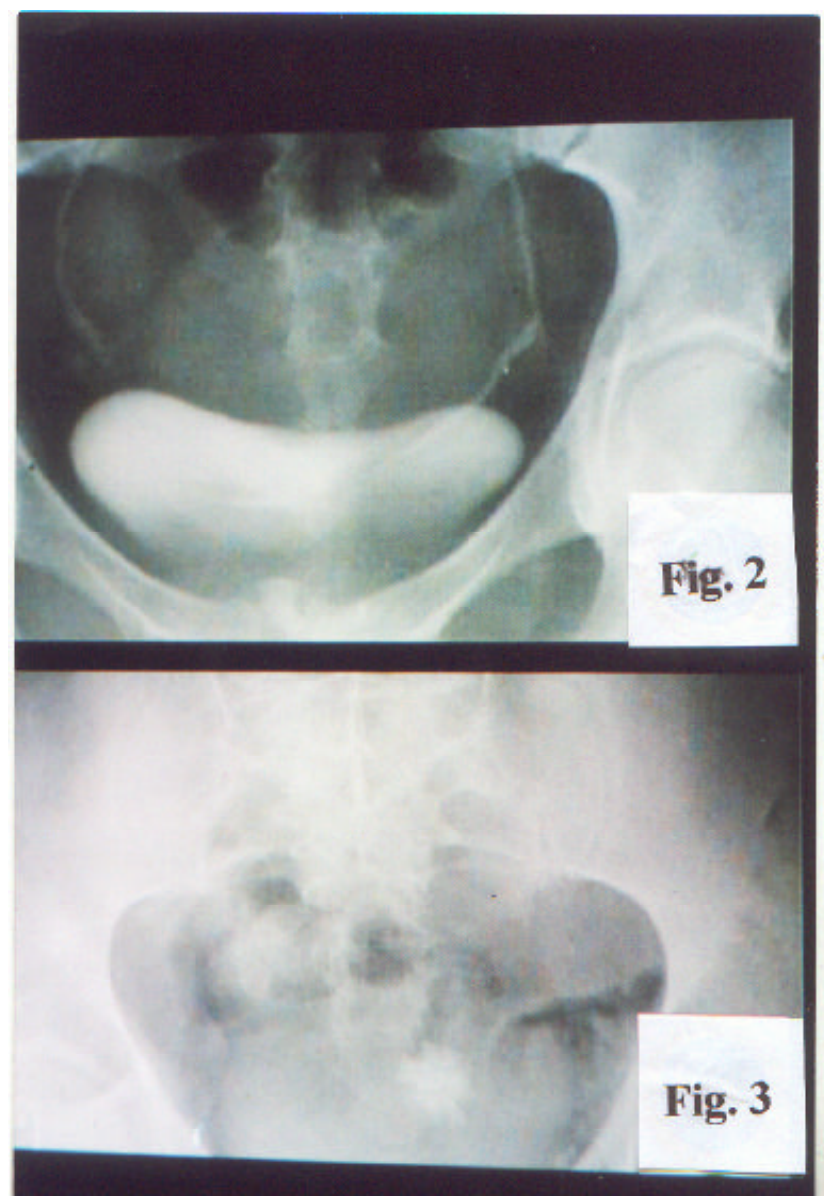

Figura 1 - Imagem radiopaca em topografia de bexiga sugerindo litíase vesical.

A conduta proposta foi tratamento clínico da infecção do trato urinário com norfloxacina durante dez dias por via oral, sendo realizado a seguir cistotomia por via abdominal, com retirada de três cálculos vesicais de aproximadamente três centímetros de diâmetro cada, que se encontravam impactados no trígono vesical. Após intervalo de três meses, procedeu-se ao reparo cirúrgico da fistula por via vaginal. A técnica cirúrgica empregada pode ser assim descrita: paciente em posição de litotomia; realização de cateterismo suprapúbico; identificação da fístula com ressecção do trajeto fistuloso, retirando-se todo tecido fibrosado até que as margens ficassem livres; fechamento das paredes vaginal e vesical em dois planos, com suturas perpendiculares entre si e interposição de peritônio entre esses planos. A paciente recebeu antibióticos no pós-operatório e o cateter vesical foi retirado no décimo quarto dia. A paciente recebeu alta hospitalar com orientações quanto a abstinência sexual por no míni- 
mo três meses e quimioprofilaxia para infecção do trato urinário.

Foi realizada cistoscopia de controle três meses após a cirurgia, cujo padrão foi normal. A paciente recebeu quimioprofilaxia com ácido nalidixico por seis meses.

\section{Discussão}

As fistulas vesicovaginais representam para suas portadoras uma condição médica e social de dificil aceitação e, por este motivo, grande atenção deve ser dada para resolução deste problema. Caracterizam-se pela história de perda contínua e involuntária de urina, sem relação com a posição que a paciente assume. Cerca de $62,5 \%$ das pacientes portadoras de fistulas urinárias se encontram entre a $5^{a}$ e $6^{a}$ década de vida, sendo a fistula vesicovaginal a mais freqüente entre as urinárias. A causa mais freqüente dessas fistulas em nosso meio é a histerectomia abdominal.

As fistulas vesicovaginais de etiologia traumática são raras, e dentre elas estão as causadas por litíase vesical ${ }^{5}$. Os cálculos primários da bexiga são relativamente raros e estão geralmente associados a urina estéril, já os cálculos secundários estão freqüentemente associados a estase urinária e infecção crônica do trato urinário.

As fistulas vesicovaginais por litíase vesical podem ser conseqüência de um fator extrinseco. Isto ocorre quando há deposição de sais urinários em torno de um núcleo constituído por material estranho, como fios inabsorviveis de cirurgias anteriores. Por outro lado, alguns cálculos provenientes dos ureteres podem se impactar na parede posterior da bexiga, alcançando grandes dimensões, causando isquemia e necrose do tecido local que são suficientes para formação de um trajeto fistuloso com a vagina, além de predispor o meio à infecção ${ }^{4}$. O caso relatado se associa provavelmente à segunda situação, uma vez que a paciente não referia cirurgias anteriores.

O diagnóstico foi feito basicamente por meio da anamnese e do exame clínico-ginecológico, além de exames complementares como o teste com azul de metileno e radiografia pélvica. A literatura aponta como métodos propedêuticos auxiliares a radiografia simples da pelve $(90 \%$ dos cálculos são radiopacos), além da urografia excretora e cistoscopia ${ }^{4}$.

A abordagem terapêutica proposta foi inicialmente clínica, visando o controle da infecção do trato urinário, com posterior tratamento cirúrgico em dois tempos, ou seja, cistotomia com exérese dos cálculos e, após intervalo de três me- ses, correção da fístula por via vaginal. Todavia, alguns estudos recomendam que não se aguarde tanto tempo para se fazer o tratamento cirúrgico e que este seja realizado logo que haja condição ideal dos tecidos lesados ${ }^{7}$.

Várias técnicas têm sido desenvolvidas para reparar cirurgicamente as fistulas vesicovaginais, porém a técnica utilizada para cada caso deve ser aquela com a qual o cirurgião tenha alcançado resultados satisfatórios ${ }^{8-11}$.

Ayhan et al. ${ }^{12}$ fazem referência aos princípios essenciais no manejo cirúrgico das fístulas genitais, enumerados por Sims em 1949, os quais são: boa visualização e adequada exposição da lesão, sutura sem tensão e apropriado estado nutricional do paciente. Os autores afirmam que esses principios devem ser rigorosamente seguidos para se obter bom êxito cirúrgico.

Estudos recentes têm questionado os méritos da correção tardia ou imediata desta fistulas, bem como da utilização das vias de reparo abdominal ou vaginal ${ }^{12-15}$.

Diaz Calleja et al. ${ }^{16}$ relataram excelentes resultados na intervenção cirúrgica de seis pacientes com fistula vesicovaginal mediante abordagem abdominal-transvesical, sendo uma delas decorrente de litíase vesical múltipla. Stovsky et al. ${ }^{17}$ referem sucesso com utilização da fulguração transuretral em fistulas relativamente pequenas ${ }^{16}$. Mckay ${ }^{18}$ relatou o tratamento de um caso de fistula vesicovaginal e vesicocutânea usando a via transuretral para cistorrafia, referindo este autor ser esta via não indicada para os casos nos quais as fistulas são extensas, associadas a anormalidades como obstrução uretral ou em casos com a possibilidade de uretra estenótica.

Preconiza-se neste serviço que a correção das fístulas jamais ocorra na fase de instalação das mesmas. Recomendamos a espera de três a quatro meses contados a partir da instalação da fístula para que se proceda à correção cirúrgica, a fim de que o trajeto fistuloso se defina e a fibrose substitua o tecido friável local.

\section{ABSTRACT}

The fistulas caused by mechanical trauma are rare, mainly the intrinsic type caused by vesical lithiasis. The treatment of these fistulas is controversial, concerning the ideal surgical technique. Several techniques have been developed, including the transvaginal and the transabdominal surgical approaches. The authors report the case of a patient with urinary loss for six months. Physical and radiological examination showed the occurrence of a vesicovaginal fistula caused by vesical lithiasis. The treatment was in two stages: 
first the extraction of the vesical stone by transabdominal approach and second, the correction of the fistula by transvaginal approach.

KEYWORDS: Fistula. Vesicovaginal fistula. Bladder stones.

\section{Referências}

1. Jones HW III, Wentz AC, Burnett LS. Novak: tratado de ginecologia. $12^{\mathrm{a}}$ ed. Rio de Janeiro: Guanabara Koogan; 1998. p.336-47.

2. Halbe HW. Tratado de ginecologia. $2^{\mathrm{a}}$ ed. São Paulo: Rocca; 1993. p.551-73.

3. Derry DE. Note on five pelves of women of eleventh dynasty in Egypty. J Obstet Gynaecol Br Emp 1935; 42:490-5.

4. Counseller US, Welch JS. Classification and etiology of genitourinary fistulae. In: Youssef AF, editor. Gynecological Urology. $1^{\text {st }}$ ed. Springfield: Thomas; 1960. p.223-7.

5. Santos JA. Tratamento cirúrgico das fístulas urogenitais: experiência de 190 casos [tese]. Rio de Janeiro: Univ. Federal do Rio de Janeiro; 1976.

6. FEBRASGO. Tratado de ginecologia. $1^{\mathrm{a}}$ ed. Rio de Janeiro: Revinter; 2000. p.1420.

7. Raz S, Bregg KJ, Nitti VW, Sussman E. Transvaginal repair of vesicovaginal fistula using a peritoneal flap. J Urol 1993; 150:56-9.

8. Tanagho EA. Urologia geral. $13^{\mathrm{a}}$ ed. Rio de Janeiro: Guanabara Koogan; 1994. p.225-6.
9. Gerber GS, Schoenberg HW. Female urinary tract fistulas. J Urol 1993; 149:229-36.

10.Elkins TE, Drescher C, Martey JO, Fort D. Vesicovaginal fistula revisited. Obstet Gynecol 1988; 72:307-12.

11.Drutz HP. Urinary fistulas. Obstet Gynecol Clin North Am 1989; 16:911-21.

12.Ayhan A, Tuncer ZS, Dogan L, Pekin S, Kisnisci HA. Results of treatment in 182 consecutive patients with genital fistulas. Int J Gynaecol Obstet 1995; 48:43-7.

13.Blaivas JG, Heritz DM, Romanzi LJ. Early versus late repair of vesicovaginal fistulas: vaginal and abdominal approaches. J Urol 1995; 153:1110-3.

14.Blandy JP, Badenoch DF, Fowler CG, Jenkins BJ, Thomas NW. Early repair of iatrogenic injury to the ureter or bladder after gynecological surgery. J Urol $1991 ; 146: 761-5$

15.Zimmern PE, Hadley HR, Staskin DR, Raz S. Genitourinary fistulae. Vaginal approach for repair of vesicovaginal fistulae. Urol Clin North Am 1985; $12: 361-7$

16.Diaz Calleja E, Calatrava Gadea S, Caldentey Garcia M, Moreno Perez F, Lapuerta Torres E, Garcia Victor F. Corrección quirúrgica de fistulas vésico-vaginales mediante abordaje abdominal-transvesical. Comentarios a esta técnica y resultados a largo plazo. Arch Esp Urol 1997; 50:55-60.

17.Stovsky MD, Ignataff JM, Blum MD, Nanninga JB, O' Connor VJ, Kursh ED. Use of electrocoagulation in the treatment of vesicovaginal fistulas. J Urol 1994; 152:1443-4.

18. McKay HA. Vesicovaginal and vesicocutaneaus fistulas: transurethral suture cystorrhaphy as a new closure technique. J Urol 1997; 158:1513-6.

Recebido em: 22/2/2001

Aceito com modificações em: 21/1/2003

\section{Bulário}

*Dados de estudos isolados, não são dados de estudos comparativos diretos $\quad$ **1035 pacientes do estudo MORE em alto risco para desenvolver doença cardiovascular

Referências Bibliográficas: 1. A.R. Genazzani, M Gambacciani. Hormone replacement therapy: the respectives for the 21st century. Maturitas 32 (1999): 11-17. 2. Bruce Ettinger, et al. Reduction of Vertebral Fracture Risk in Postmenopausal Women With Osteoporosis Treated With Raloxifene. Results From a 3-Year Randomized Clinical Trial. JAMA 1999; 282:637-645. 3. Michael R. McClung, et al. Effect of Risedronate on the Risk of Hip Fracture in Elderly Women. N Eng J Med 2001; 344:333-40. 4. Dennis M. Black, et al. Randomized trial of alendronate on risk of fracture in women with existing vertebral fractures. Lancet 1996; 358: 1535-41. 5. Chesnut CH, et al. Am J Med. 2000;109:267-276. 6. Ettinger B, et al. JAMA. 1999;282:637-645; \& data on file, Eli Lilly and Company. 7. Harris ST, et al. JAMA. 1999;282:1344-1352. 8. Reginster J-Y, et al. Osteoporosis Int. 2000;11:83-91. 9. Black J Med. 2000;109:267-276. 6. Ettinger B, et al. JAMA. 1999;282:637-645; \& data on file, Eli Lilly and Company. 7. Harris ST, et al. JAMA. 1999;282:1344-1352. 8. Reginster J-Y, et al. Osteoporosis Int. 2000;11:83-91. 9. Black
DM, et al. Lancet. 1996;348:1535-1541. 10. Cummings SR, et al. JAMA. 1998:280:2077-2082. 11. Michael Maricic, et al. Early effects of Raloxifene on Clinical Vertebral Fractures at 12 Months in Postmenopausal Women with
Osteoporosis. Arch Intern Med 2002; 162:1140-1143. 12. Siris et al. Effects Raloxifene on Fracture Severity in Postmenopausal Women with Osteoporosis: Results from the MORE Study. Osteoporos Int (2002) 13:907-913. 13. R. Eastell, et al.The Effects of Raloxifene on Incident Vertebral Fractures in Postmenopausal Women with Osteoporosis: 4-Year Results from the MORE Trial. ASBMR 22nd Annual Meeting. J Bone Miner Res 2000; 15 (suppl 1): S229. 14. Elizabeth Barret-Conor, et al. Raloxifene and Cardiovascular Events in Osteoporotic Postmenopausal Women. Four -Year Results from the MORE randomized Trial. JAMA 2002; 287:847-857. 15. Jane A.Cauley, et al. Continued breast cancer risk reduction in postmenopausal women treated with raloxifene: 4-year results from the MORE trial. Breast Cancer Research and Treatment 65: 125-134, 2001. 16. Pierre D. Delmas, et al. Effects of Raloxifene on Bone Mineral Densty, Serum Cholesterol Concentrations, and Uterine Endometrium in Postmenopausal Women. N Eng J Med 1997; 337:1641-7.

EVISTA (Cloridrato de Raloxifeno) - Informaç̃os para Prescrição:
Indicações - EVISTA® é indicado para o tratamento e prevenção da osteoporose em mulheres após a menopausa. Contra-Indicaç̃os - EVISTA® é contra-indicado em mulheres que estão ou podem ficar grávidas, pacientes com história atual ou pregressa de episódios tromboembólicos venosos e pacientes com hipersensibilidade ao raloxifeno ou aos excipientes do comprimido. Advertências - Raloxifeno está associado com um risco maior de desenvolver episódio tromboembólico que é semelhante ao risco relacionado com a terapia de reposição hormonal. O tratamento com EVISTA® deve ser interrompido no caso de doença ou condição que leve a um período prolongado de imobilização. O uso de EVISTA® não é recomendado para pacientes com insuficiência hepática. Precaucọes - Não se recomenda o uso concomitante de EVISTA® com estrógenos sistêmicos. Raloxifeno não é eficaz na redução de manifestações vasomotoras (rubores, calores ou ondas de calor) associada com deficiência de estrógeno. USO DURANTE A GRAVIDEZ E LACTAÇÃO - EVISTA® só deve ser usado em mulheres pós-menopausadas. Não deve ser tomado por mulheres com potencial para engravidar ou mulheres lactantes. EVISTA® pode afetar o desenvolvimento fetal. Interações Medicamentosas - Se o raloxifeno for administrado junto com varfarina ou outro derivado cumarínico, o tempo de protrombina deve ser monitorizado. Raloxifeno não deve ser administrado junto com colestiramina. Durante o tratamento com EVISTA® comumente são observadas as seguintes alterações séricas : aumento da subfração HDL-2 do colesterol e da apolipoproteína A1 e redução no colesterol total, LDL colesterol, fibrinogênio, apolipoproteína B e lipoproteína(a). Reações Adversas Episódios tromboembólicos venosos (trombose venosa profunda, embolia pulmonar e trombose da veia retiniana), tromboflebite venosa superficial, vasodilatação (fogachos), cãibras nas pernas, edema periférico, contagem plaquetária ligeiramente diminuída, sintomas de gripe. Posologia e Administração - A posologia recomendada é um comprimido de EVISTA® (60 mg de cloridrato de raloxifeno) uma vez ao dia administrado por via oral podendo ser tomado a qualquer hora do dia independente das refeições. VENDA SOB PRESCRIÇAO MÉDICA. Para maiores informações, consulte a bula completa do produto ou o Serviço de Atendimento ao Cliente Lilly SAC 0800 7010444, e-mail: sac_brasil@lilly.com. Caixa Postal 21.313 .6 CEP $04602-970$ - São Paulo. 